\title{
On Ulam Stability of a Generalized Delayed Differential Equation of Fractional Order
}

\author{
Janusz Brzdek@i), Nasrin Eghbali, and Vida Kalvandi
}

\begin{abstract}
We investigate Ulam stability of a general delayed differential equation of a fractional order. We provide formulas showing how to generate the exact solutions of the equation using functions that satisfy it only approximately. Namely, the approximate solution $\phi$ generates the exact solution as a pointwise limit of the sequence $\Lambda^{n} \phi$ with some integral (possibly, nonlinear) operator $\Lambda$. We estimate the speed of convergence and the distance between those approximate and exact solutions. Moreover, we provide some exemplary calculations, involving the Chebyshev and Bielecki norms and some semigauges, that could help to obtain reasonable outcomes for such estimations in some particular cases. The main tool is the Diaz-Margolis fixed point alternative.
\end{abstract}

Mathematics Subject Classification. 26A33, 34D10, 39B82, 45M10, 47H10.

Keywords. Fractional order derivative, delayed differential equation, Ulam stability, fixed point, semigauge, Chebyshev norm, Bielecki norm.

\section{Introduction}

The differential equations of a fractional order have numerous applications in the modeling of various physical phenomena and processes in economics, chemistry, aerodynamics, etc. (for further information see [12-19,21]). They are also an excellent tool for the description of hereditary properties of many materials. For details concerning the fractional calculus we refer to $[12,15,19$, $20]$.

Unfortunately, quite often we only have a description of approximate solutions to such equations and it is very difficult to get any sufficient information on the exact solutions to them. So, the natural question is how big 
is the difference between those approximate and exact solutions and whether it is possible to generate somehow the exact solutions by the means of those approximate ones. Some convenient tools to study such issues provides the theory of Ulam (often also called the Hyers-Ulam) type stability. It has been motivated by a problem of Ulam, concerning approximate homomorphisms of groups, and an answer to it provided by Hyers [6] (see [3,7,11,22] for more details and references).

The main idea of such stability can be very roughly expressed in the following way: When a function satisfying an equation approximately (in some sense) must be near an exact solution to the equation?

The following definition (cf. [3, p. 119, Ch. 5, Definition 8]) makes that notion a bit more precise $\left(\mathbb{R}_{+}\right.$stands for the set of all nonnegative reals and $C^{D}$ denotes the family of all functions mapping a set $D \neq \emptyset$ into a set $C \neq \emptyset$ ).

Definition 1. Let $A$ be a nonempty set, $(X, d)$ be a metric space, $\mathcal{E} \subset \mathcal{C} \subset \mathbb{R}_{+}{ }^{A}$ be nonempty, $\mathcal{T}$ be an operator mapping $\mathcal{C}$ into $\mathbb{R}_{+}{ }^{A}$ and $\mathcal{F}_{1}, \mathcal{F}_{2}$ be operators mapping nonempty set $\mathcal{D} \subset X^{A}$ into $X^{A}$. We say that the equation

$$
\mathcal{F}_{1} \varphi(x)=\mathcal{F}_{2} \varphi(x)
$$

is $\left(\mathcal{E}, \mathcal{T}\right.$ - -stable provided for any $\varepsilon \in \mathcal{E}$ and $\varphi_{0} \in \mathcal{D}$ with

$$
d\left(\mathcal{F}_{1} \varphi_{0}(x), \mathcal{F}_{2} \varphi_{0}(x)\right) \leq \varepsilon(x), \quad x \in A
$$

there exists a solution $\varphi \in \mathcal{D}$ of Eq. (1) such that

$$
d\left(\varphi(x), \varphi_{0}(x)\right) \leq \mathcal{T} \varepsilon(x), \quad x \in A .
$$

Roughly speaking, $(\mathcal{E}, \mathcal{T})$ - stability of (1) means that every approximate (in the sense of (2)) solution of (1) is always close (in the sense of (3)) to an exact solution of (1). For some recent results in this area, concerning the fractional-order differential equations, see [8-10,18,23-30]. We continue those investigations for Eq. (4) given a bit later.

In what follows, $\mathbb{R}$ stands for the field of real numbers. Moreover, for every positive integer $n, C^{n}\left(D_{1}, D_{2}\right)$ always denotes (as usual) the family of all functions from a real interval $D_{1}$ into a real interval $D_{2}$, that are $n$-times continuously differentiable. Analogously, $C\left(D_{1}, D_{2}\right)$ means the family of all functions from an interval $D_{1} \subset \mathbb{R}$ into an interval $D_{2} \subset \mathbb{R}$, that are continuous.

\section{Preliminaries}

The derivatives of a fractional order can be defined in various ways, but we only use the Caputo fractional derivative, which in a simplified way will be denoted by $D^{\alpha}$ for $\alpha \in \mathbb{R}_{+}$. Below we remind the definition of it ( $\Gamma$ denotes the usual Gamma function, and $[\alpha]$ means the integer part of a real number $\alpha$, i.e., $[\alpha]:=\max \{n \in \mathbb{Z}: n \leq \alpha\}$, where $\mathbb{Z}$ is the set of integers). 
Definition 2. Let $\alpha \in \mathbb{R}, n=[\alpha]+1$ and $D$ be a real interval with $-\infty<d:=$ $\min D$. The Caputo fractional derivative, of order $\alpha$, is given by

$$
D^{\alpha} \eta(s)={ }^{c} D_{d^{+}}^{\alpha} \eta(s)=\frac{1}{\Gamma(n-\alpha)} \int_{d}^{s} \frac{\eta^{(n)}(r) d r}{(s-r)^{\alpha+1-n}}, \quad s \in D,
$$

for every function $\eta \in C^{n}(D, \mathbb{R})$.

The advantage of the Caputo derivative is that (contrary to the RiemannLiouville fractional derivative) it does not involve any initial conditions of fractional order while solving differential equations including it.

In this paper, $\alpha \in(0,1)$ and $h>0$ are fixed, $I \subset \mathbb{R}$ is an interval, which has one of the following three forms: $\left[t_{0}, a\right),\left[t_{0}, a\right],\left[t_{0}, \infty\right.$ ) (for some $\left.a, t_{0} \in \mathbb{R}, a>t_{0}\right), H:=[-h, 0], H_{t_{0}}:=\left[t_{0}-h, t_{0}\right]$ and $I^{h}:=I \cup H_{t_{0}}$. Next, $\xi: I^{h} \rightarrow I^{h}, \omega: H_{t_{0}} \rightarrow \mathbb{R}$ and $f: I \times \mathbb{R} \times C(H, \mathbb{R}) \rightarrow \mathbb{R}$ are fixed functions satisfying appropriate regularity conditions (specified later).

If $y \in C\left(I^{h}, \mathbb{R}\right)$ and $t \in I$, then we define the function $y_{t} \in C(H, \mathbb{R})$ by

$$
y_{t}(\tau)=y(t+\tau), \quad \tau \in H
$$

We consider approximate solutions $y \in C^{1}\left(I^{h}, \mathbb{R}\right)$ for the following general delayed fractional differential equation

$$
D^{\alpha}(y(s) g(s))=f\left(s, y(\xi(s)), y_{s}\right), \quad s \in I, \quad y(s)=\omega(s), \quad s \in H_{t_{0}},
$$

with a fixed function $g \in C^{1}\left(I^{h}, \mathbb{R}\right)$. That is we study functions $y \in C^{1}\left(I^{h}, \mathbb{R}\right)$ satisfying the inequality

$$
\begin{aligned}
& \left|D^{\alpha}[y(s) g(s)]-f\left(s, y(\xi(s)), y_{s}\right)\right| \leq \Phi(s), \quad s \in I, \\
& y(s)=\omega(s), \quad s \in H_{t_{0}},
\end{aligned}
$$

with a given function $\Phi: I \rightarrow \mathbb{R}_{+}$satisfying some natural restrictions.

Solutions to a particular case of (4) have been investigated in [13] for

$$
g(t)=e^{\gamma t}, \quad f\left(t, y(t), y_{t}\right)=f_{0}\left(t, y_{t}\right) e^{\gamma t},
$$

with a fixed real number $\gamma>0$ and the function $f_{0}: I \times C(H, \mathbb{R}) \rightarrow \mathbb{R}$, satisfying some regularity conditions. In such a case Eq. (4) is equivalent (cf. $[5,13])$ to the following integral equation

$$
\begin{aligned}
& y(s)=y\left(t_{0}\right) e^{\gamma\left(t_{0}-s\right)}+\frac{1}{\Gamma(\alpha)} \int_{t_{0}}^{s} \frac{f_{0}\left(r, y_{r}\right) d r}{(s-r)^{1-\alpha} e^{\gamma(s-r)}}, \quad s \in I, \\
& y(s)=\omega(s), \quad s \in H_{t_{0}} .
\end{aligned}
$$

We refer to [13] for results on solutions to (6).

In [1] the authors investigated Ulam's type stability of a simplified version of (4) with the Chebyshev (supremum) norm $\|\cdot\|_{\infty}$ in $C(H, \mathbb{R})$. Namely, the following outcome has been proved in $\left[1\right.$, Theorem 2.4] $\left(\mathbb{R}_{+}^{0}\right.$ stands for the set of positive reals). 
Theorem 1. Let $\tau_{0} \in \mathbb{R}_{+}, \kappa_{0} \in(0,1)$, functions $f_{1}: I \times C(H, \mathbb{R}) \rightarrow \mathbb{R}, \lambda: I \rightarrow$ $\mathbb{R}_{+}$and $\Phi: I^{h} \rightarrow \mathbb{R}_{+}^{0}$ be continuous, $g \in C^{1}\left(I^{h}, \mathbb{R}\right)$ be fixed, and the following three inequalities be valid:

$$
\begin{aligned}
& \left|f_{1}\left(s, v_{s}\right)-f_{1}\left(s, u_{s}\right)\right| \leq \lambda(s)\left\|v_{s}-u_{s}\right\|_{\infty}, \quad v, u \in C\left(I^{h}, \mathbb{R}\right), s \in I, \\
& \frac{1}{\Gamma(\alpha) g(s)} \int_{t_{0}}^{s} \frac{\Phi(r) d r}{(s-r)^{1-\alpha}} \leq \tau_{0} \Phi(s), \quad s \in I, \\
& \frac{1}{\Gamma(\alpha) g(s)} \int_{t_{0}}^{s} \frac{\lambda(r) \Phi_{h}(r) d r}{(s-r)^{1-\alpha}} \leq \kappa_{0} \Phi(s), \quad s \in I,
\end{aligned}
$$

where $\Phi_{h}(r):=\sup _{t \in[r-h, r]} \Phi(t)$. Let $y \in C^{1}\left(I^{h}, \mathbb{R}\right)$ be such that

$$
\begin{aligned}
& \left|D^{\alpha}[y(s) g(s)]-f_{1}\left(s, y_{s}\right)\right| \leq \Phi(s), \quad s \in I, \\
& y(s)=\omega(s), \quad s \in H_{t_{0}} .
\end{aligned}
$$

Then there exists a unique function $\widehat{y} \in C^{1}\left(I^{h}, \mathbb{R}\right)$ with

$$
\begin{aligned}
D^{\alpha}[\widehat{y}(s) g(s)] & =f_{1}\left(s, \widehat{y}_{s}\right), \quad s \in I, & \widehat{y}(s)=\omega(s), \quad s \in H_{t_{0}}, \\
|y(s)-\widehat{y}(s)| \leq\left(1-\kappa_{0}\right)^{-1} \tau_{0} \Phi(s), & & s \in I .
\end{aligned}
$$

Please note that no regularity conditions on $\omega$ in Theorem 1 have been assumed explicitly, but actually from the assumption that $y$ is continuously differentiable and (11) it follows that $\omega$ must be continuously differentiable.

In this paper we present a significant generalization of Theorem 1, because we consider Eq. (4), which is much more general than (12). Moreover, we admit a wider range of ways of measuring the distance in $C(H, \mathbb{R})$. Namely, instead of the supremum norm as in (7), we use semigauges depicted in the subsequent Definition 3 (cf., e.g., [2]), which include the cases of various norms, seminorms, quasinorms, semi-quasinorms etc. We provide some suitable examples at the end of this paper.

Furthermore, we provide formulas showing how to generate the exact solutions of the equation from the functions that satisfy it only approximately. Namely, some approximate solutions $\phi$ of (4) generate the exact solutions as the pointwise limits of the sequence $\Lambda^{n} \phi$ with an integral operator $\Lambda$, given by (26). We estimate the speed of this convergence and the distance between $\phi$ and that generated exact solution. In the second part of the paper, we provide some exemplary calculations, involving in particular the Chebyshev and Bielecki norms, that could help to obtain reasonable outcomes for such estimations in several particular cases.

Let us introduce the following definition (cf., e.g., $[2,3])$.

Definition 3. Let $A$ be a real linear space. A function $\rho: A \rightarrow[0,+\infty]$ (i.e., $\rho$ may take the infinite value $+\infty)$ is a semigauge on $A$ if $\rho(0)=0$ and

$$
\rho(\lambda x)=|\lambda| \rho(x), \quad \lambda \in \mathbb{R}, x \in A, \rho(x)<\infty .
$$

A gauge on $A$ is any semigauge $\rho$ on $A$ such that $\rho(x) \neq 0$ for $x \neq 0$. 
Note that if $\rho$ is a semigauge on a real linear space $A, x \in A$ and $\rho(\lambda x)<$ $\infty$ for some $\lambda \in \mathbb{R} \backslash\{0\}$, then $\rho(\eta x)<\infty$ for every $\eta \in \mathbb{R}$, because (13) yields $\rho(\eta x)=\left|\eta \lambda^{-1}\right| \rho(\lambda x)$.

Remark 1. Clearly, the norms and extended norms are gauges. Let us recall that an extended norm, on a real (or complex) linear space $X$, is a function $\|\cdot\|: X \rightarrow[0,+\infty]$ (i.e., $\|\cdot\|$ may also take the value $+\infty$ ) such that, for every scalar $\alpha$ and every $x, y \in X$ with $\|x\|,\|y\| \in[0,+\infty)$,

$$
\|x+y\| \leq\|x\|+\|y\|, \quad\|\alpha x\|=|\alpha|\|x\|,
$$

and the equality $\|x\|=0$ holds if and only if $x$ is the zero vector.

If $Y$ is a real or complex normed space and $S$ is a nonempty set, then an extended norm in $Y^{S}$ can be defined by:

$$
\|f\|=\sup _{s \in S}\|f(s)\|, \quad f \in Y^{S} .
$$

Further, if $f$ is a linear functional on a real or complex linear space $X$, then the formula:

$$
\rho(x)=|f(x)|, \quad x \in X,
$$

defines a semigauge on $X$.

Finally, let us recall the Diaz-Margolis fixed point alternative (see [4]), which will be useful in the proof of our main result. To this end we need the following definition.

Definition 4. An extended metric on a set $X \neq \emptyset$ is a function $d: X^{2} \rightarrow$ $[0,+\infty]$ satisfying the following three conditions:

(1) $d(x, y)=0$ if and only if $x=y$;

(2) $d(x, y)=d(y, x)$ for all $x, y \in X$

(3) $d(x, z) \leq d(x, y)+d(y, z)$ for all $x, y, z \in X$.

In the sequel, given a set $X \neq \emptyset$ and $\mathcal{L}: X \rightarrow X$, we sometimes write for simplicity $\mathcal{L} x:=\mathcal{L}(x)$ for $x \in X$. Moreover, as usual, $\mathcal{L}^{0} x:=x$ and $\mathcal{L}^{n} x:=\mathcal{L}\left(\mathcal{L}^{n-1} x\right)$ for $x \in X, n \in \mathbb{N}$ (positive integers). The Diaz-Margolis fixed point alternative [4] can be formulated as follows $\left(\mathbb{N}_{0}:=\mathbb{N} \cup\{0\}\right)$.

Theorem 2. Let $d$ be an extended complete metric on a nonempty set $X$ and $\mathcal{L}: X \rightarrow X$ be contractive with the Lipschitz constant $L<1$ (i.e., $d(\mathcal{L} x, \mathcal{L} y) \leq$ $L d(x, y)$ for $x, y \in X$ with $d(x, y) \in(0,+\infty))$. Assume that $x \in X$ is such that there exists $k \in \mathbb{N}$ with $d\left(\mathcal{L}^{k-1} x, \mathcal{L}^{k} x\right)<\infty$. Then the sequence $\left(\mathcal{L}^{n} x\right)_{n \in \mathbb{N}}$ converges to a fixed point $x^{*} \in X$ of $\mathcal{L}, x^{*}$ is the unique fixed point of $\mathcal{L}$ in the set $X^{*}=\left\{y \in X: d\left(x^{*}, y\right)<\infty\right\}$ and

$$
d\left(\mathcal{L}^{n} x, x^{*}\right) \leq \frac{L^{n-k+1} d\left(\mathcal{L}^{k-1} x, \mathcal{L}^{k} x\right)}{1-L}, \quad n \in \mathbb{N}_{0}, n \geq k-1
$$


Proof. The convergence of $\mathcal{L}^{n} x$ to a fixed point $x^{*}$ of $\mathcal{L}$ results from [4, Theorem]. The uniqueness of $x^{*}$ follows from the subsequent simple inequality

$$
d\left(u, x^{*}\right)=d\left(\mathcal{L}^{n} u, \mathcal{L}^{n} x^{*}\right) \leq L^{n} d\left(u, x^{*}\right), \quad n \in \mathbb{N},
$$

which is true for every fixed point $u \in X^{*}$ of $\mathcal{L}$.

Also the proof of (14) is a routine, but for the convenience of readers, we present it. So, note that, for each $m \in \mathbb{N}, m \geq k$,

$$
\begin{aligned}
d\left(\mathcal{L}^{k-1} x, \mathcal{L}^{m} x\right) & \leq \sum_{i=k}^{m} d\left(\mathcal{L}^{i-1} x, \mathcal{L}^{i} x\right) \\
& \leq d\left(\mathcal{L}^{k-1} x, \mathcal{L}^{k} x\right) \sum_{i=0}^{m-k} L^{i} \leq \frac{d\left(\mathcal{L}^{k-1} x, \mathcal{L}^{k} x\right)}{1-L}
\end{aligned}
$$

and consequently

$$
\begin{aligned}
d\left(\mathcal{L}^{k-1} x, x^{*}\right) & \leq d\left(\mathcal{L}^{k-1} x, \mathcal{L}^{m} x\right)+d\left(\mathcal{L}^{m} x, x^{*}\right) \\
& \leq \frac{d\left(\mathcal{L}^{k-1} x, \mathcal{L}^{k} x\right)}{1-L}+d\left(\mathcal{L}^{m} x, x^{*}\right) .
\end{aligned}
$$

Since $\lim _{m \rightarrow \infty} d\left(\mathcal{L}^{m} x, x^{*}\right)=0$, this implies that

$$
d\left(\mathcal{L}^{k-1} x, x^{*}\right) \leq \frac{d\left(\mathcal{L}^{k-1} x, \mathcal{L}^{k} x\right)}{1-L} .
$$

Now (14) results from the above inequality and from the fact that, for every $n \in \mathbb{N}_{0}$ with $n \geq k-1$,

$$
d\left(\mathcal{L}^{n} x, x^{*}\right)=d\left(\mathcal{L}^{n-k+1}\left(\mathcal{L}^{k-1} x\right), \mathcal{L}^{n-k+1} x^{*}\right) \leq L^{n-k+1} d\left(\mathcal{L}^{k-1} x, x^{*}\right) .
$$

Remark 2. Assume that $k=1$ in Theorem 2. Clearly, in such a case, (14) (with $n=0$ ) implies that $d\left(x, x^{*}\right)<+\infty$, whence $x \in X^{*}$. Let $z \in X$ be a fixed point of $\mathcal{L}$ with $d(x, z)<+\infty$. Then $d\left(x^{*}, z\right) \leq d\left(x^{*}, x\right)+d(x, z)<+\infty$ and consequently $z \in X^{*}$, which means that $z=x^{*}$, because $x^{*}$ is the unique fixed point of $\mathcal{L}$ in $X^{*}$.

So, if there is a fixed point $z \in X \backslash\left\{x^{*}\right\}$, then $d(x, z)=+\infty$.

\section{The Main Result}

In the sequel, $\rho$ is a semigauge on $C(H, \mathbb{R}), \mathcal{U} \subset C\left(I, \mathbb{R}_{+}\right)$is nonempty and $\mathcal{G}: \mathcal{U} \rightarrow \mathbb{R}_{+}^{I}$ is given. Next, $\xi: I^{h} \rightarrow I^{h}$ and $f: I \times \mathbb{R} \times C(H, \mathbb{R}) \rightarrow \mathbb{R}$ are such that the function $f_{w}: I \rightarrow \mathbb{R}_{+}$, given by:

$$
f_{w}(s)=f\left(s, w(\xi(s)), w_{s}\right), \quad s \in I,
$$

is continuous for every $w \in C\left(I^{h}, \mathbb{R}\right)$. 
Moreover, we assume that there is a nondecreasing sequence $\left(r_{n}\right)_{n \in \mathbb{N}}$ in $I$ such that

$$
I=\bigcup_{n \in \mathbb{N}}\left[t_{0}, r_{n}\right], \quad \xi\left(\left[t_{0}, r_{n}\right]\right) \subset\left[t_{0}, r_{n}\right], \quad n \in \mathbb{N} .
$$

Remark 3. Clearly, if $\xi(t) \leq t$ for $t \in I$, then any nondecreasing sequence $\left(r_{n}\right)_{n \in \mathbb{N}}$ in $I$, with $\sup _{n \in \mathbb{N}} r_{n}=\sup I$, fulfils (16). Moreover, if $I=\left[t_{0}, b\right]$ with some $b>t_{0}$, then (16) holds with the constant sequence $r_{n}:=b$ for $n \in \mathbb{N}$.

We write

$$
I_{n}:=\left[t_{0}, r_{n}\right], \quad I_{n}^{h}:=\left[t_{0}-h, r_{n}\right], \quad n \in \mathbb{N} .
$$

Next, $g: I \rightarrow \mathbb{R} \backslash\{0\}$ is an arbitrarily fixed function that is continuously differentiable. We define $\mathcal{T}: C\left(I, \mathbb{R}_{+}\right) \rightarrow C\left(I, \mathbb{R}_{+}\right)$by

$$
(\mathcal{T} \chi)(t):=\frac{1}{\Gamma(\alpha)|g(t)|} \int_{t_{0}}^{t} \frac{\chi(s)}{(t-s)^{1-\alpha}} d s, \quad t \in I, \chi \in C_{I}\left(\mathbb{R}_{+}\right) .
$$

We also need the following two hypotheses (with $n \in \mathbb{N}$ ) concerning functions $\Phi \in \mathcal{U}$.

$\left(\mathcal{H}_{n}\right)$ If $w \in C\left(I_{n}^{h}, \mathbb{R}\right)$ is such that $|w(s)| \leq \Phi(s)$ for $s \in I_{n}$ and $w(s)=0$ for $s \in H_{t_{0}}$, then $\rho\left(w_{t}\right) \leq(\mathcal{G} \Phi)(t)$ for every $t \in I_{n}$.

$(\mathcal{H})$ There is a continuous function $L: I \rightarrow \mathbb{R}_{+}$with $(\mathcal{T} \Phi)(t) \leq L(t) \Phi(t)$ for $t \in I$.

Examples of functions $\Phi$ satisfying the hypotheses, with suitable operators $\mathcal{G}$ (cf., e.g., (47)), are given in the further parts of the paper.

Remark 4. If the semigauge $\rho$ is nondecreasing, i.e., when $\rho\left(\phi_{1}\right) \leq \rho\left(\phi_{2}\right)$ for every $\phi_{1}, \phi_{2} \in C(H, \mathbb{R})$ with $\left|\phi_{1}(t)\right| \leq\left|\phi_{2}(t)\right|$ for every $t \in H$, then $\left(\mathcal{H}_{n}\right)$ holds for each $n \in \mathbb{N}$ with $(\mathcal{G} \Phi)(t)=\rho\left(\Phi^{t}\right)$ for $t \in I$ and $\Phi \in \mathcal{U}$, where $\Phi^{t}: H \rightarrow \mathbb{R}$ is given by

$$
\Phi^{t}(s)= \begin{cases}\Phi(t+s), & \text { if } s \geq t_{0}-t \\ \Phi\left(t_{0}\right), & \text { otherwise }\end{cases}
$$

Unfortunately not all semigauges on $C(H, \mathbb{R})$ must be nondecreasing. For instance, it is easily seen that the semigauge $\rho$ given by

$$
\rho(\phi)=\sup _{s \in H}|\phi(s)|-\inf _{s \in H}|\phi(s)|, \quad \phi \in C(H, \mathbb{R}),
$$

is not nondecreasing, because it equals zero on each constant function.

Some further examples of semigauges, both nondecreasing and not, can be found in Sect. 6 .

Now we have all tools to prove the next theorem, which is the main result of this paper. 
Theorem 3. Let $\Phi \in \mathcal{U}$ fulfil hypotheses $(\mathcal{H})$ and $\left(\mathcal{H}_{n}\right)$ for all $n \in \mathbb{N}$. Let $\lambda: I \times$ $\mathbb{R}_{+}^{2} \rightarrow \mathbb{R}_{+}$be such that the functions $\lambda(x, \cdot, v)$ and $\lambda(x, v, \cdot)$ are nondecreasing for every $x \in I$ and $v \in \mathbb{R}_{+}$, the function

$$
I \ni s \rightarrow \lambda(s, \mu \Phi(\xi(s)), \mu(\mathcal{G} \Phi)(s)) \in \mathbb{R}_{+}
$$

is locally integrable for each $\mu \in \mathbb{R}_{+}^{0}$, and there is a function $K: I \rightarrow[0,1)$ with

$$
\frac{1}{\Gamma(\alpha)|g(t)|} \int_{t_{0}}^{t} \frac{\lambda(s, \mu \Phi(\xi(s)), \mu(\mathcal{G} \Phi)(s))}{(t-s)^{1-\alpha} \mu} d s \leq K(t) \Phi(t), t \in I, \mu \in \mathbb{R}_{+}^{0} \text {. }
$$

Assume also that

$$
\begin{gathered}
\left|f\left(t, s_{1}, z_{t}\right)-f\left(t, s_{2}, w_{t}\right)\right| \leq \lambda\left(t,\left|s_{1}-s_{2}\right|, \rho\left(z_{t}-w_{t}\right)\right), \\
t \in I, s_{1}, s_{2} \in \mathbb{R}, z, w \in C\left(I^{h}, \mathbb{R}\right), \rho\left(z_{t}-w_{t}\right)<\infty,
\end{gathered}
$$

and $K$ is continuous or nondecreasing.

If $y \in C^{1}\left(I^{h}, \mathbb{R}\right)$ is such that

$$
\begin{aligned}
& \left|D^{\alpha}[y(s) g(s)]-f\left(s, y(\xi(s)), y_{s}\right)\right| \leq \Phi(s), \quad s \in I, \\
& y(s)=\omega(s), \quad s \in H_{t_{0}},
\end{aligned}
$$

then there is a unique function $\widehat{y} \in C^{1}\left(I^{h}, \mathbb{R}\right)$, which fulfils the conditions

$$
\begin{aligned}
& D^{\alpha}[\widehat{y}(s) g(s)]=f\left(s, \widehat{y}(\xi(s)), \widehat{y}_{s}\right), \quad s \in I, \quad \widehat{y}(s)=\omega(s), \quad s \in H_{t_{0}}, \\
& |y(s)-\widehat{y}(s)| \leq \frac{(\mathcal{T} \Phi)(s)}{1-K_{n}}, \quad s \in I_{n}, n \in \mathbb{N},
\end{aligned}
$$

where $K_{n}:=\sup _{t \in I_{n}} K(t)$ for $n \in \mathbb{N}$. Moreover,

$$
\begin{aligned}
& \widehat{y}(s)=\lim _{k \rightarrow \infty}\left(\Lambda^{k} y\right)(s), \quad s \in I^{h}, \\
& \left|\widehat{y}(s)-\left(\Lambda^{k} y\right)(s)\right| \leq \frac{K_{n}^{k}(\mathcal{T} \Phi)(s)}{1-K_{n}}, \quad s \in I_{n}, n, k \in \mathbb{N},
\end{aligned}
$$

where, for each $w \in C\left(I^{h}, \mathbb{R}\right), \Lambda: C\left(I^{h}, \mathbb{R}\right) \rightarrow C\left(I^{h}, \mathbb{R}\right)$ is given by the formula

$$
\begin{aligned}
& (\Lambda w)(s)=w\left(t_{0}\right) \frac{g\left(t_{0}\right)}{g(s)}+\frac{1}{\Gamma(\alpha) g(s)} \int_{t_{0}}^{s} \frac{f\left(r, w(\xi(r)), w_{r}\right)}{(s-r)^{1-\alpha}} d r, \quad s \in I, \\
& (\Lambda w)(s)=\omega(s), \quad s \in H_{t_{0}} .
\end{aligned}
$$

Proof. Let $y \in C^{1}\left(I^{h}, \mathbb{R}\right)$ fulfil $(21)$. Fix $n \in \mathbb{N}$ and denote by $X_{n}$ the space of all continuous functions $w: I_{n}^{h} \rightarrow \mathbb{R}$ such that $w(t)=\omega(t)$ for $t \in H_{t_{0}}$. Define an extended complete metric in $X_{n}$ by $d_{n}(z, w)=\inf \left\{C \in[0, \infty):|z(t)-w(t)| \leq C \Phi(t)\right.$ for $\left.t \in I_{n}\right\}, \quad z, w \in X_{n}$.

The metric may take the infinite value $+\infty$ (i.e., can be an extended metric), because we do not exclude the situation where $\Phi(t)=0$ for some $t \in I_{n}$ and assume that $\inf \emptyset=\infty$. The completeness of $d_{n}$ follows from the fact that $\Phi$ is continuous on $I_{n}$ and therefore bounded, which means that the 
convergence in $X_{n}$, with respect to $d_{n}$, is actually the uniform convergence on $I_{n}$ (with regard to the natural distance in $\mathbb{R}$ ).

Let $p \in(1, \infty)$ be such that $(1-\alpha) p<1$ and $q:=p /(p-1)$. Then $1 / p+1 / q=1$ and $p(\alpha-1)+1>0$. Since for every $w \in C\left(I^{h}, \mathbb{R}\right)$ the function $f_{w}$ (defined by (15)) is continuous, the functions

$$
I \ni s \rightarrow\left|f_{w}(s)\right|^{q}, \quad I \ni s \rightarrow f_{w}(s)(t-s)^{p(\alpha-1)}
$$

are continuous and, by the Hölder inequality, we have

$$
\left|\int_{t_{0}}^{t} \frac{f_{w}(s) d s}{(t-s)^{1-\alpha}}\right| \leq\left(\int_{t_{0}}^{t}\left|f_{w}(s)\right|^{q} d s\right)^{1 / q}\left(\int_{t_{0}}^{t}(t-s)^{p(\alpha-1)} d s\right)^{1 / p}<+\infty
$$

for every $t \in I$, whence the improper integral

$$
\int_{t_{0}}^{t} \frac{f\left(s, w(\xi(s)), w_{s}\right) d s}{(t-s)^{1-\alpha}}
$$

is convergent for every $t \in I$. This means that we can define operator $\Lambda_{n}$ : $X_{n} \rightarrow X_{n}$ by the formula

$$
\begin{aligned}
& \left(\Lambda_{n} w\right)(t)=w\left(t_{0}\right) \frac{g\left(t_{0}\right)}{g(t)}+\frac{1}{\Gamma(\alpha) g(t)} \int_{t_{0}}^{t} \frac{f\left(s, w(\xi(s)), w_{s}\right) d s}{(t-s)^{1-\alpha}}, \quad t \in I_{n}, \\
& \left(\Lambda_{n} w\right)(t)=\omega(t), \quad t \in H_{t_{0}}
\end{aligned}
$$

for each $w \in X_{n}$ (see (16)).

Take $z, w \in X_{n}$ with $C_{z w}:=d(z, w)<+\infty$, which means

$$
|z(t)-w(t)| \leq C_{z w} \Phi(t), \quad t \in I_{n} .
$$

Write

$$
\widetilde{z}:=\frac{1}{C_{z w}} z, \quad \widetilde{w}:=\frac{1}{C_{z w}} w .
$$

Since $|\widetilde{z}(t)-\widetilde{w}(t)| \leq \Phi(t)$ for $t \in I_{n}$, by $\left(\mathcal{H}_{n}\right)$, we have

$$
\rho\left(\widetilde{z}_{t}-\widetilde{w}_{t}\right) \leq(\mathcal{G} \Phi)(t), \quad t \in I_{n}
$$

and consequently

$$
\rho\left(z_{t}-w_{t}\right)=\rho\left(C_{z w} \widetilde{z}_{t}-C_{z w} \widetilde{w}_{t}\right) \leq C_{z w} \rho\left(\widetilde{z}_{t}-\widetilde{w}_{t}\right) \leq C_{z w}(\mathcal{G} \Phi)(t), \quad t \in I_{n} .
$$

So, (20) and the monotonicity assumptions on $\lambda$ yield

$$
\begin{aligned}
\left|f\left(t, z(\xi(t)), z_{t}\right)-f\left(t, w(\xi(t)), w_{t}\right)\right| & \leq \lambda\left(t,|z(\xi(t))-w(\xi(t))|, \rho\left(z_{t}-w_{t}\right)\right) \\
& \leq \lambda\left(t, C_{z w} \Phi(\xi(t)), C_{z w}(\mathcal{G} \Phi)(t)\right), \quad t \in I_{n}
\end{aligned}
$$


whence (19) implies that

$$
\begin{aligned}
\left|\left(\Lambda_{n} z\right)(t)-\left(\Lambda_{n} w\right)(t)\right| & \leq \frac{1}{\Gamma(\alpha)|g(t)|} \int_{t_{0}}^{t} \frac{\left|f\left(s, z(\xi(s)), z_{s}\right)-f\left(s, w(\xi(s)), w_{s}\right)\right|}{(t-s)^{1-\alpha}} d s \\
& \leq \frac{1}{\Gamma(\alpha)|g(t)|} \int_{t_{0}}^{t} \frac{\lambda\left(s, C_{z w} \Phi(\xi(s)), C_{z w}(\mathcal{G} \Phi)(s)\right)}{(t-s)^{1-\alpha}} d s \\
& \leq C_{z w} K(t) \Phi(t) \leq K_{n} C_{z w} \Phi(t), \quad t \in I_{n},
\end{aligned}
$$

because $z\left(t_{0}\right)=\omega\left(t_{0}\right)=w\left(t_{0}\right)$. This means that

$$
d\left(\Lambda_{n} z, \Lambda_{n} w\right) \leq K_{n} C_{z w} .
$$

Since $K$ is continuous or nondecreasing, so $K_{n}<1$ and consequently $\Lambda_{n}$ is contractive on $X_{n}$. Next, integrating the inequality in (21) from $t_{0}$ to $t$, we get

$$
\begin{gathered}
\left|y(t) g(t)-y\left(t_{0}\right) g\left(t_{0}\right)-\frac{1}{\Gamma(\alpha)} \int_{t_{0}}^{t} \frac{f\left(s, y(\xi(s)), y_{s}\right)}{(t-s)^{1-\alpha}} d s\right| \\
\quad=\left|\frac{1}{\Gamma(\alpha)} \int_{t_{0}}^{t} \frac{D^{\alpha}[\widehat{y}(t) g(t)]-f\left(s, y(\xi(s)), y_{s}\right)}{(t-s)^{1-\alpha}} d s\right| \\
\quad \leq \frac{1}{\Gamma(\alpha)} \int_{t_{0}}^{t} \frac{\left|D^{\alpha}[\widehat{y}(t) g(t)]-f\left(s, y(\xi(s)), y_{s}\right)\right|}{(t-s)^{1-\alpha}} d s \\
\leq \frac{1}{\Gamma(\alpha)} \int_{t_{0}}^{t} \frac{\Phi(s)}{(t-s)^{1-\alpha}} d s, \quad t \in I,
\end{gathered}
$$

SO

$$
\begin{gathered}
\left|y(t)-y\left(t_{0}\right) \frac{g\left(t_{0}\right)}{g(t)}-\frac{1}{\Gamma(\alpha) g(t)} \int_{t_{0}}^{t} \frac{f\left(s, y(\xi(s)), y_{s}\right)}{(t-s)^{1-\alpha}} d s\right| \\
\leq \frac{1}{\Gamma(\alpha)|g(t)|} \int_{t_{0}}^{t} \frac{\Phi(s)}{(t-s)^{1-\alpha}} d s=(\mathcal{T} \Phi)(t), \quad t \in I,
\end{gathered}
$$

whence in view of hypothesis $(\mathcal{H})$

$$
\left|y_{n}(t)-\left(\Lambda_{n} y_{n}\right)(t)\right| \leq(\mathcal{T} \Phi)(t) \leq L(t) \Phi(t), \quad t \in I_{n},
$$

where $y_{n}(t)=y(t)$ for $t \in I_{n}$. That is we have the inequality:

$$
d\left(y_{n}, \Lambda_{n} y_{n}\right) \leq L_{n}:=\sup _{t \in I_{n}} L(t)<+\infty .
$$

Consequently, by Theorem 2 (with $k=1, \mathcal{L}=\Lambda_{n}$ and $L=K_{n}$ ) and Remark 2 , there is a unique fixed point $\widehat{y}_{n} \in C\left(I_{n}^{h}, \mathbb{R}\right)$ of $\Lambda_{n}$ with $d\left(\widehat{y}_{n}, y_{n}\right)<$ $\infty$,

$$
\widehat{y}_{n}(t)=\lim _{k \rightarrow \infty}\left(\Lambda_{n}^{k} y_{n}\right)(t), \quad t \in I_{n}^{h}
$$

and

$$
d\left(\widehat{y}_{n}, \Lambda_{n}^{m} y_{n}\right) \leq \frac{K_{n}^{m} d\left(\Lambda_{n} y_{n}, y_{n}\right)}{1-K_{n}}, \quad m \in \mathbb{N}_{0}
$$


Clearly, $\widehat{y}_{n}$ as a fixed point of $\Lambda_{n}$ is continuously differentiable on the interval $\left[t_{0}, t_{n}\right)$. Further, since the uniqueness means in this case that

$$
\widehat{y}_{n}(t)=\widehat{y}_{l}(t), \quad n, l \in \mathbb{N}, l \leq n, t \in I_{l},
$$

we can define $\widehat{y} \in C\left(I^{h}, \mathbb{R}\right)$ by

$$
\widehat{y}(t):=\widehat{y}_{n}(t), \quad t \in I_{n}, n \in \mathbb{N} .
$$

It is easily seen that $\widehat{y}$ is continuously differentiable and it is the unique solution to (22) fulfilling

$$
|\widehat{y}(t)-y(t)| \leq \frac{d\left(\Lambda_{n} y_{n}(t), y_{n}(t)\right)}{1-K_{n}} \leq \frac{(\mathcal{T} \Phi)(t)}{1-K_{n}}, \quad t \in I_{n}, n \in \mathbb{N},
$$

because of the uniqueness of $\widehat{y}_{n}$ and the definition of $\Lambda_{n}$.

Finally, notice that (28) and (29) imply (24).

Remark 5. Assume that $\xi(t) \leq t$ for each $t \in I$. Then the points $r_{n}$ can be selected quite arbitrarily and, for a given $t \in I$, we can assume that $t=r_{j}$ with some $j \in \mathbb{N}$ (see Remark 3). As $\widehat{y}$ in Theorem 3 is unique and its form (given by (24)) in this situation does not depend on the choice of points $r_{n}$, this means that, for such $\xi$, (23) can be actually replaced by the following inequality

$$
|y(t)-\widehat{y}(t)| \leq \frac{(\mathcal{T} \Phi)(t)}{1-\bar{K}(t)}, \quad t \in I
$$

where $\bar{K}(t):=\sup _{s \in\left[t_{0}, t\right]} K(s)$ for $t \in I$. Clearly, if $K$ is nondecreasing, then $\bar{K}=K$.

Remark 6. Assume that $\Phi(t) \neq 0$ for each $t \in I$. Then hypothesis $(\mathcal{H})$ holds with $L$ given by

$$
L(t)=\frac{(\mathcal{T} \Phi)(t)}{\Phi(t)}, \quad t \in I
$$

Remark \%. It can be very difficult to calculate precisely the form of the function $\mathcal{T} \Phi$ occurring in (23) and (25). Therefore we show below some very easy approaches that might help to obtain some useful estimations of it.

Let $t \in I, m \in \mathbb{N}, m>1$, and $s_{1}, \ldots, s_{m} \in I$ be such that $s_{1}:=t_{0}<$ $s_{2}<\ldots<s_{m}:=t$. Write $J_{i}:=\left[s_{i}, s_{i+1}\right]$ for $i=1, \ldots, m-1$. Then it is easily seen that

$$
(\mathcal{T} \Phi)(t)=\sum_{k=1}^{m-1} \frac{1}{\Gamma(\alpha) g(t)} \int_{J_{k}}(t-s)^{\alpha-1} \Phi(s) d s
$$


and we have the subsequent two estimations:

$$
\begin{aligned}
& \sum_{k=1}^{m-2} \frac{\left(t-s_{k+1}\right)^{\alpha-1}}{\Gamma(\alpha) g(t)} \int_{J_{k}} \Phi(s) d s \leq \sum_{k=1}^{m-2} \frac{\left(t-s_{k+1}\right)^{\alpha-1} \Phi_{k}}{\Gamma(\alpha) g(t)}, \\
(\mathcal{T} \Phi)(t) & \leq \sum_{k=1}^{m-1} \frac{\Phi_{k}}{\Gamma(\alpha) g(t)} \int_{J_{k}}(t-s)^{\alpha-1} d s \\
& \leq \sum_{k=1}^{m-1} \frac{\Phi_{k}\left(\left(t-s_{k}\right)^{\alpha}-\left(t-s_{k+1}\right)^{\alpha}\right)}{\Gamma(\alpha+1) g(t)}
\end{aligned}
$$

where $\Phi_{k}:=\sup _{s \in J_{k}} \Phi(s)$ for $k=1, \ldots, m-1$. Also, if $m>2$, then (32) implies that

$$
(\mathcal{T} \Phi)(t) \leq \sum_{k=1}^{m-2} \frac{\left(t-s_{k+1}\right)^{\alpha-1}}{\Gamma(\alpha) g(t)} \int_{J_{k}} \Phi(s) d s+\frac{\Phi_{m-1}\left(t-s_{m-1}\right)^{\alpha}}{\Gamma(\alpha+1) g(t)}
$$

If $\Phi$ is nondecreasing, then $\Phi_{k}=\Phi\left(s_{k+1}\right)$ for $k=1, \ldots, m-1$ and (34) yields the inequality

$$
(\mathcal{T} \Phi)(t) \leq \sum_{k=1}^{m-1} \frac{\Phi\left(s_{k+1}\right)\left(\left(t-s_{k}\right)^{\alpha}-\left(t-s_{k+1}\right)^{\alpha}\right)}{\Gamma(\alpha+1) g(t)}
$$

whence for $m=2$ we get the following very simple form

$$
(\mathcal{T} \Phi)(t) \leq \frac{\Phi(t)\left(t-t_{0}\right)^{\alpha}}{\Gamma(\alpha+1) g(t)}
$$

while for $m=3$ we have

$$
\begin{aligned}
\Gamma(\alpha) g(t)(\mathcal{T} \Phi)(t) & \leq \Phi\left(s_{2}\right) \int_{t_{0}}^{s_{2}}(t-s)^{\alpha-1} d s+\Phi(t) \int_{s_{2}}^{t}(t-s)^{\alpha-1} d s \\
& \leq \frac{\left(s_{2}-t_{0}\right) \Phi\left(s_{2}\right)}{s_{2}^{1-\alpha}}+\frac{\Phi(t)\left(t-s_{2}\right)^{\alpha}}{\alpha}
\end{aligned}
$$

which gives the estimation

$$
(\mathcal{T} \Phi)(t) \leq \frac{1}{\Gamma(\alpha) g(t)} \inf _{s \in\left(t_{0}, t\right)}\left(\frac{\left(s-t_{0}\right) \Phi(s)}{s^{1-\alpha}}+\frac{(t-s)^{\alpha} \Phi(t)}{\alpha}\right) .
$$

Clearly, we can argue analogously when $\Phi$ is nonincreasing, because then $\Phi_{k}=\Phi\left(s_{k}\right)$. Also, we can choose for estimations (34) and (35) points $s_{k}$ such that $\Phi$ is nondecreasing or nonincreasing on each interval $J_{k}$. 
Note also that we can use the following Hölder type inequality:

$$
\begin{aligned}
(\mathcal{T} \Phi)(t) & =\frac{1}{\Gamma(\alpha) g(t)} \int_{t_{0}}^{t}(t-s)^{\alpha-1} \Phi(s) d s \\
& \leq \frac{1}{\Gamma(\alpha) g(t)}\left(\int_{t_{0}}^{t}(t-s)^{p(\alpha-1)} d s\right)^{1 / p}\left(\int_{t_{0}}^{t}(\Phi(s))^{q} d s\right)^{1 / q} \\
& =\frac{\left(t-t_{0}\right)^{\alpha-1+1 / p}}{(p \alpha-p+1) \Gamma(\alpha) g(t)}\left(\int_{t_{0}}^{t}(\Phi(s))^{q} d s\right)^{1 / q}, \quad t \in I
\end{aligned}
$$

for any $p, q \in(1, \infty)$ such that $1 / p+1 / q=1$ and $p(1-\alpha)<1$.

Clearly, we may also combine those approaches, applying different estimations in different intervals $J_{k}$ and for different values of $t$.

\section{Consequence of Theorem 3}

Now, we show how to use Theorem 3 to easily generate solutions of some differential equations of fractional order. Namely, let $\Phi \in \mathcal{U}, \lambda: I \times \mathbb{R}_{+}^{2} \rightarrow \mathbb{R}_{+}$ and $K: I \rightarrow[0,1)$ be as in Theorem 3. Let $\bar{f}: I \times \mathbb{R} \times C(H, \mathbb{R}) \rightarrow \mathbb{R}$ be such that

$$
\begin{aligned}
& \left|\bar{f}\left(t, s_{1}, z_{t}\right)-\bar{f}\left(t, s_{2}, w_{t}\right)\right| \leq \lambda\left(t,\left|s_{1}-s_{2}\right|, \rho\left(z_{t}-w_{t}\right)\right), \\
& \quad t \in I, z, w \in C\left(I^{h}, \mathbb{R}\right), s_{1}, s_{2} \in \mathbb{R}
\end{aligned}
$$

and the function $\bar{f}_{w}: I \rightarrow \mathbb{R}_{+}$is continuous for every $w \in C\left(I^{h}, \mathbb{R}\right)$, where

$$
\bar{f}_{w}(s):=\bar{f}\left(s, w(\xi(s)), w_{s}\right), \quad s \in I .
$$

We have the following corollary.

Corollary 1. Let $y \in C^{1}\left(I^{h}, \mathbb{R}\right), y(s)=\omega(s)$ for $s \in H_{t_{0}}$, and

$$
\Psi(t):=D^{\alpha}[y(t) g(t)]-\bar{f}\left(t, y(\xi(t)), y_{t}\right), \quad t \in I .
$$

Then, for each function $\Delta \in C_{I}(\mathbb{R})$ with $|\Psi(t)-\Delta(t)| \leq \Phi(t)$ for $t \in I$, there is a unique $\widehat{y} \in C^{1}\left(I^{h}, \mathbb{R}\right)$ satisfying the conditions

$$
\begin{aligned}
& D^{\alpha}[\widehat{y}(t) g(t)]=\bar{f}\left(t, \widehat{y}(\xi(t)), \widehat{y}_{t}\right)+\Delta(t), \quad t \in I, \\
& \widehat{y}(s)=\omega(s), \quad s \in H_{t_{0}},
\end{aligned}
$$

such that

$$
|y(t)-\widehat{y}(t)| \leq \frac{(\mathcal{T} \Phi)(t)}{1-K_{n}}, \quad t \in I_{n}, n \in \mathbb{N}
$$


where $K_{n}:=\sup _{t \in I_{n}} K(t)$ for $n \in \mathbb{N}$. Moreover,

$$
\begin{aligned}
& \widehat{y}(t)=\lim _{k \rightarrow \infty}\left(\Lambda_{0}^{k} y\right)(t), \quad t \in I^{h}, \\
& \left|\widehat{y}(t)-\left(\Lambda_{0}^{k} y\right)(t)\right| \leq \frac{K_{n}^{k}(\mathcal{T} \Phi)(t)}{1-K_{n}}, \quad t \in I_{n}, n, k \in \mathbb{N},
\end{aligned}
$$

where, for each $w \in C\left(I^{h}, \mathbb{R}\right), \Lambda_{0}: C\left(I^{h}, \mathbb{R}\right) \rightarrow C\left(I^{h}, \mathbb{R}\right)$ is given by the formula

$$
\begin{aligned}
& \left(\Lambda_{0} w\right)(t)=w\left(t_{0}\right) \frac{g\left(t_{0}\right)}{g(t)}+\frac{1}{\Gamma(\alpha) g(t)} \int_{t_{0}}^{t} \frac{\bar{f}\left(s, w(\xi(s)), w_{s}\right)+\Delta(t)}{(t-s)^{1-\alpha}} d s, \quad t \in I, \\
& \left(\Lambda_{0} w\right)(t)=\omega(t), \quad t \in H_{t_{0}} .
\end{aligned}
$$

Proof. Take $\Delta \in C(I, \mathbb{R})$ with $|\Psi(t)-\Delta(t)| \leq \Phi(t)$ for $t \in I$. Note that

$$
\left|D^{\alpha}[y(t) g(t)]-\bar{f}\left(t, y(\xi(t)), y_{t}\right)-\Delta(t)\right|=|\Psi(t)-\Delta(t)| \leq \Phi(t), \quad t \in I .
$$

Hence, it is enough to use Theorem 3 with $f(t, s, w)=\bar{f}(t, s, w)+\Delta(t)$ for $t \in I, s \in \mathbb{R}$ and $w \in C(H, \mathbb{R})$.

\section{The Chebyshev Norm}

Let $\rho=\|\cdot\|_{\infty}$ be the Chebyshev (supremum) norm on $C(H, \mathbb{R})$, i.e.,

$$
\rho(u):=\sup _{s \in H}|u(s)|, \quad u \in C(H, \mathbb{R}) .
$$

Then it is easily seen that, if $\mathcal{U}=C\left(I, \mathbb{R}_{+}\right)$, then (see Remark 4) hypothesis $\left(\mathcal{H}_{n}\right)$ is valid for every $n \in \mathbb{N}$ and every function $\Phi \in \mathcal{U}$ with $\mathcal{G}$ defined by the formula

$$
(\mathcal{G} \phi)(t):=\sup _{s \in I \cap[t-h, t]} \phi(s), \quad \phi \in \mathcal{U}, t \in I .
$$

Consequently, Theorem 3 implies Theorem 1; it is enough to take the functions $K$ and $L$ constant, $\xi(t) \equiv t$, and

$$
\begin{gathered}
\lambda(t, r, s):=\lambda_{0}(t) s, \quad t \in I, r, s \in \mathbb{R}_{+}, \\
f(t, s, z):=f_{1}(t, z), \quad t \in I, z \in C(H, \mathbb{R}), s \in \mathbb{R},
\end{gathered}
$$

with some continuous $\lambda_{0}: I \rightarrow \mathbb{R}_{+}$and $f_{1}: I \times C\left(I^{h}, \mathbb{R}\right) \rightarrow \mathbb{R}$.

If $\Phi \in \mathcal{U}$ is a nondecreasing function and $\mathcal{G}$ is given by (47), then it is easily seen that $\mathcal{G} \Phi=\Phi$. Next, for $\xi(t) \equiv t$ and $f$ given by

$$
f(t, r, s):=f_{0}(t)\left(a_{1} r+a_{2} s\right)+\Delta(t), \quad t \in I, r, s \in \mathbb{R}_{+},
$$

with some fixed $a_{1}, a_{2} \in \mathbb{R}$ and continuous $\Delta, f_{0}: I \rightarrow \mathbb{R}$, the function $\lambda$ can be defined by

$$
\lambda(t, r, s):=\left|f_{0}(t)\right|\left(\left|a_{1}\right| r+\left|a_{2}\right| s\right), \quad t \in I, r, s \in \mathbb{R}_{+} .
$$


For such a case we can derive from Theorem 3 and Remarks 5 and 6 the following simple result.

Corollary 2. Let $K: I \rightarrow[0,1)$ be nondecreasing, $\Phi: I \rightarrow \mathbb{R}_{+}^{0}$ be continuous and nondecreasing, and

$$
\begin{aligned}
& \left.\left|f\left(t, s_{1}, z_{t}\right)-f\left(t, s_{2}, w_{t}\right)\right| \leq\left|f_{0}(t)\right|\left(a_{1}\left|s_{1}-s_{2}\right|+a_{2} \| z_{t}-w_{t}\right) \|_{\infty}\right), \\
& \quad t \in I, z, w \in C\left(I^{h}, \mathbb{R}\right), s_{1}, s_{2} \in \mathbb{R}, \\
& \frac{a_{1}+a_{2}}{\Gamma(\alpha) g(t)} \int_{t_{0}}^{t} \frac{\left|f_{0}(s)\right| \Phi(s)}{(t-s)^{1-\alpha}} d s \leq K(t) \Phi(t), \quad t \in I .
\end{aligned}
$$

If $y \in C^{1}\left(I^{h}, \mathbb{R}\right)$ is such that

$$
\begin{aligned}
& \left|D^{\alpha}[y(t) g(t)]-f\left(t, y(t), y_{t}\right)\right| \leq \Phi(t), \quad t \in I, \\
& y(s)=\omega(s), \quad s \in H_{t_{0}},
\end{aligned}
$$

then there exists a unique function $\widehat{y} \in C^{1}\left(I^{h}, \mathbb{R}\right)$ with

$$
\begin{aligned}
& D^{\alpha}[\widehat{y}(t) g(t)]=f\left(t, \widehat{y}(t), \widehat{y}_{t}\right), \quad t \in I, \quad \widehat{y}(s)=\omega(s), \quad s \in H_{t_{0}}, \\
& |y(t)-\widehat{y}(t)| \leq \frac{(\mathcal{T} \Phi)(t)}{1-K(t)}, \quad t \in I .
\end{aligned}
$$

Moreover, (24) holds with $\Lambda: C\left(I^{h}, \mathbb{R}\right) \rightarrow C\left(I^{h}, \mathbb{R}\right)$ defined by $(26)$ and

$$
\left|\widehat{y}(t)-\left(\Lambda^{k} y\right)(t)\right| \leq \frac{K(t)^{k}(\mathcal{T} \Phi)(t)}{1-K(t)}, \quad t \in I, k \in \mathbb{N} .
$$

It is desirable to know how to determine the function $K$ occurring in (19) and (52), because of the forms of (22), (54) and (55). Certainly, we can use the methods proposed in Remark 7. Unfortunately, in some cases this can lead to quite involved calculations. For the convenience of the readers, we present below a simple observation.

Remark 8. Let $\Phi: I \rightarrow \mathbb{R}_{+}$be such that (52) holds with some $K: I \rightarrow[0,1)$. Clearly, this means that

$$
\frac{a_{1}+a_{2}}{\Phi(t) \Gamma(\alpha) g(t)} \int_{t_{0}}^{t} \frac{\left|f_{0}(s)\right| \Phi(s)}{(t-s)^{1-\alpha}} d s<1, \quad t \in I, \Phi(t) \neq 0 .
$$

Hence, in the case $0 \notin \Phi(I)$ (as in Corollary 2), we can define the function $K$ simply by

$$
K(t)=\frac{a_{1}+a_{2}}{\Phi(t) \Gamma(\alpha) g(t)} \int_{t_{0}}^{t} \frac{\left|f_{0}(s)\right| \Phi(s)}{(t-s)^{1-\alpha}} d s, \quad t \in I .
$$

Now, consider the case $0 \in \Phi(I), 0 \notin f_{0}(I)$. If $s \in I$ is such that $\Phi(s)=0$, then (52) implies that $\left[t_{0}, s\right) \subset \Phi^{-1}(\{0\})$. Hence the case $\sup \Phi^{-1}(\{0\})=\sup I$ is trivial, because $\Phi(t)=0$ for each $t \in I$ and $K$ can be any nondecreasing function mapping $I$ into $[0,1)$. 
So, assume that $t^{\prime}:=\sup \Phi^{-1}(\{0\})<\sup I$. Then $\Phi(t)=0$ for every $t \in I$ with $t \leq t^{\prime}$ and $\Phi(t) \neq 0$ for every $t \in I$ with $t>t^{\prime}$, whence it is enough to take

$$
K(t)= \begin{cases}\sup _{s \in\left[t_{0}, t\right]} \chi(s), & \text { if } t>t^{\prime}, \\ 0 & \text { if } t \leq t^{\prime}\end{cases}
$$

where

$$
\chi(t):=\frac{a_{1}+a_{2}}{\Phi(t) \Gamma(\alpha) g(t)} \int_{t_{0}}^{t} \frac{\left|f_{0}(s)\right| \Phi(s)}{(t-s)^{1-\alpha}} d s, \quad t \in I .
$$

The value of the integral

$$
\int_{t_{0}}^{t} \frac{\left|f_{0}(s)\right| \Phi(s)}{(t-s)^{1-\alpha}} d s
$$

can be estimated with the methods proposed in Remark 7 .

\section{Nondecreasing Semigauges}

In this last section of the paper we discuss some possible forms of semigauges.

Assume that semigauge $\rho$ is nondecreasing. As we have noticed in Remark 4, then we can take $(\mathcal{G} \Phi)(t)=\rho\left(\Phi^{t}\right)$ for $t \in I$ and $\Phi \in \mathcal{U}$, where $\Phi^{t}: H \rightarrow \mathbb{R}$ is given by (18). Consequently, condition (19) has the following form

$$
\frac{1}{\Gamma(\alpha)|g(t)|} \int_{t_{0}}^{t} \frac{\lambda\left(s, \mu \Phi(\xi(s)), \mu \rho\left(\Phi^{s}\right)\right)}{(t-s)^{1-\alpha} \mu} d s \leq K(t) \Phi(t), \quad t \in I, \mu \in \mathbb{R}_{+}^{0} .
$$

Next, if $\xi(t) \equiv t, f$ is given by (49) with some fixed $a_{1}, a_{2} \in \mathbb{R}$ and continuous $\Delta, f_{0}: I \rightarrow \mathbb{R}$, and $\lambda$ is defined by (50), then (56) simplifies further to

$$
\frac{1}{\Gamma(\alpha)|g(t)|} \int_{t_{0}}^{t} \frac{\left|f_{0}(s)\right|\left(\left|a_{1}\right| \Phi(s)+\left|a_{2}\right| \rho\left(\Phi^{s}\right)\right)}{(t-s)^{1-\alpha}} d s \leq K(t) \Phi(t), \quad t \in I .
$$

Now, we will give some examples of nondecreasing semigauges $\rho$. To this end fix continuous functions $\Delta, f_{1}, f_{2}: I \rightarrow \mathbb{R}$ and functions $A_{1}, A_{2}: \mathbb{R} \rightarrow \mathbb{R}$ satisfying the following Lipschitz conditions

$$
\left|A_{i}(s)-A_{i}(t)\right| \leq b_{i}|s-t|, \quad s, t \in \mathbb{R}, i=1,2,
$$

with some fixed $b_{1}, b_{2} \in \mathbb{R}_{+}$. Define $f$ and $\lambda$ by

$$
\begin{aligned}
f(t, r, w) & :=f_{1}(t) A_{1}(r)+f_{2}(t) A_{2}(\mathcal{J}(w))+\Delta(t), t \in I, r \in \mathbb{R}, w \in C(H, \mathbb{R}), \\
\lambda(t, r, s) & :=\left|f_{1}(t)\right| b_{1} r+\left|f_{2}(t)\right| b_{2} s, \quad t \in I, r, s \in \mathbb{R}_{+},
\end{aligned}
$$

where $\mathcal{J}: C(H, \mathbb{R}) \rightarrow \mathbb{R}$ is given and will be depicted a little later (according to the form of $\rho$ ). 
First, take $\kappa: H \rightarrow \mathbb{R}$ and consider the case where $\rho$ has the Bielecki-type form, that is

$$
\rho(w)=\sup _{t \in H}|\kappa(t) w(t)|, \quad w \in C(H, \mathbb{R})
$$

Clearly, if $\kappa(t) \equiv e^{-\gamma t}$ with some $\gamma>0$, then $\rho$ is the Bielecki norm $\|\cdot\|_{B}$, given by

$$
\|w\|_{B}=\sup _{t \in H} e^{-\gamma t}|w(t)|, \quad w \in C(H, \mathbb{R}) .
$$

Note that, with such $\rho$ (and $\lambda$ given by (59)), condition (20) holds for $f$ defined by (58), e.g., with $\mathcal{J} \in\left\{\mathcal{J}_{1}, \ldots, \mathcal{J}_{11}\right\}$, where $\mathcal{J}_{1}, \ldots, \mathcal{J}_{11}: C(H, \mathbb{R}) \rightarrow$ $\mathbb{R}$ are given by

$$
\begin{aligned}
& \mathcal{J}_{1}(w)=d+\frac{1}{h} \int_{-h}^{0} \kappa(s) w(s) d s, \quad \mathcal{J}_{2}(w)=d+\frac{1}{h} \int_{-h}^{0} \kappa(s)|w(s)| d s, \\
& \mathcal{J}_{3}(w)=d+\rho(w), \quad \mathcal{J}_{4}(w)=d+\sup _{s \in H} \kappa(s) w(s), \\
& \mathcal{J}_{5}(w)=d+\sup _{s \in H} \kappa(s)|w(s)|, \quad \mathcal{J}_{6}(w)=d+\inf _{s \in H}|\kappa(s) w(s)|, \\
& \mathcal{J}_{7}(w)=d+\inf _{s \in H} \kappa(s)|w(s)|, \quad \mathcal{J}_{8}(w)=d+\inf _{s \in H} \kappa(s) w(s), \\
& \mathcal{J}_{9}(w)=\sum_{k=1}^{8} d_{k}\left|\mathcal{J}_{k}(w)\right|, \quad \mathcal{J}_{10}(w)=\sum_{k=1}^{8} d_{k} \mathcal{J}_{k}(w), \\
& \mathcal{J}_{11}(w)=\sum_{k=1}^{8}\left|d_{k} \mathcal{J}_{k}(w)\right|
\end{aligned}
$$

with fixed $d, d_{1}, \ldots, d_{8} \in \mathbb{R}$ such that $\sum_{k=1}^{8}\left|d_{k}\right| \leq 1$ (in the case of $\mathcal{J}_{1}$ and $\mathcal{J}_{2}$ we assume that $\kappa$ is locally integrable and in any other case $\kappa$ should have adequate regularity for the integral in (56) to exist).

If $\kappa$ is locally integrable and $\rho$ has the form

$$
\rho(w)=\frac{1}{h} \int_{-h}^{0} \kappa(s)|w(s)| d s, \quad w \in C(H, \mathbb{R}),
$$

then condition (20) holds for $f$ and $\lambda$ defined by (58) and (59), e.g., with $\mathcal{J} \in\left\{\mathcal{J}_{1}, \mathcal{J}_{2}\right\}$, where $\mathcal{J}_{1}$ and $\mathcal{J}_{2}$ are given as above. We also can define $\rho$ by

$$
\rho(w)=\left|\frac{1}{h} \int_{-h}^{0} \kappa(s) w(s) d s\right|, \quad w \in C(H, \mathbb{R}),
$$

and for such $\rho$ condition (20) holds for $f$ and $\lambda$ defined by (58) and (59), with $\mathcal{J}=\mathcal{J}_{1}$, where $\mathcal{J}_{1}$ is again given as above. In this situation estimation (20) is finer than with $\rho$ given by (60), but it is easily seen that (61) defines a semigauge that is not nondecreasing. 
Next, fix $m \in \mathbb{N}, s_{1}, \ldots, s_{m} \in H$ and $c_{1}, \ldots, c_{m}, d \in \mathbb{R}$. If

$$
\rho(w)=\sum_{k=1}^{m}\left|c_{i} w\left(s_{k}\right)\right|, \quad w \in C(H, \mathbb{R}),
$$

then condition (20) holds for $f$ and $\lambda$ defined by (58) and (59), with $\mathcal{J} \in$ $\left\{\mathcal{J}_{1}, \ldots, \mathcal{J}_{4}\right\}$, where for all $w \in C(H, \mathbb{R})$,

$$
\begin{aligned}
& \mathcal{J}_{1}(w)=d+\rho(w), \\
& \mathcal{J}_{2}(w)=d+\sum_{k=1}^{m} c_{i} w\left(s_{k}\right), \\
& \mathcal{J}_{3}(w)=d+\sum_{k=1}^{m} c_{i}\left|w\left(s_{k}\right)\right|, \\
& \mathcal{J}_{4}(w)=d+\left|\sum_{k=1}^{m} c_{i} w\left(s_{k}\right)\right| .
\end{aligned}
$$

We also can define $\rho$ by the formula

$$
\rho(w)=\left|\sum_{k=1}^{m} c_{i} w\left(s_{k}\right)\right|, \quad w \in C(H, \mathbb{R}),
$$

and then (20) holds for $f$ and $\lambda$ defined by (58) and (59), e.g., with $\mathcal{J}$ : $C(H, \mathbb{R}) \rightarrow \mathbb{R}$ given by

$$
\mathcal{J}(w)=d+\sum_{k=1}^{m} c_{i} w\left(s_{k}\right), \quad w \in C(H, \mathbb{R}) ;
$$

however, such semigauge $\rho$ is not nondecreasing.

If

$$
\rho(w)=\max _{k=1, \ldots, m}\left|c_{i} w\left(s_{k}\right)\right|, \quad w \in C(H, \mathbb{R}),
$$

then condition (20) holds for $f$ and $\lambda$ defined by (58) and (59), e.g., with $\mathcal{J} \in\left\{\mathcal{J}_{1}, \ldots, \mathcal{J}_{6}\right\}$, where

$$
\begin{array}{lll}
\mathcal{J}_{1}(w)=d+\rho_{\kappa}(w), & \mathcal{J}_{2}(w)=d+\max _{k=1, \ldots, m} c_{i} w\left(s_{k}\right), \\
\mathcal{J}_{3}(w)=d+\max _{k=1, \ldots, m} c_{i}\left|w\left(s_{k}\right)\right|, & \mathcal{J}_{4}(w)=d+\min _{k=1, \ldots, m} c_{i} w\left(s_{k}\right), \\
\mathcal{J}_{5}(w)=d+\min _{k=1, \ldots, m}\left|c_{i} w\left(s_{k}\right)\right|, & \mathcal{J}_{6}(w)=d+\min _{k=1, \ldots, m} c_{i}\left|w\left(s_{k}\right)\right|
\end{array}
$$

for all $w \in C(H, \mathbb{R})$.

The semigauge $\rho$ can also be defined by the formula

$$
\rho_{m}(w)=\left|\max _{k=1, \ldots, m} c_{i} w\left(s_{k}\right)\right|, \quad w \in C(H, \mathbb{R}),
$$

and then (20) holds for $f$ and $\lambda$ defined by (58) and (59), with $\mathcal{J}: C(H, \mathbb{R}) \rightarrow \mathbb{R}$ given by

$$
\mathcal{J}(w)=d+\max _{k=1, \ldots, m} c_{i} w\left(s_{k}\right), \quad w \in C(H, \mathbb{R})
$$


however, $\rho$ does not have to be nondecreasing then.

\section{Declarations}

Conflict of interest The authors declare that they have no conflict of interest.

Open Access. This article is licensed under a Creative Commons Attribution 4.0 International License, which permits use, sharing, adaptation, distribution and reproduction in any medium or format, as long as you give appropriate credit to the original author(s) and the source, provide a link to the Creative Commons licence, and indicate if changes were made. The images or other third party material in this article are included in the article's Creative Commons licence, unless indicated otherwise in a credit line to the material. If material is not included in the article's Creative Commons licence and your intended use is not permitted by statutory regulation or exceeds the permitted use, you will need to obtain permission directly from the copyright holder. To view a copy of this licence, visit http://creativecommons. org/licenses/by/4.0/.

\section{References}

[1] Brzdek, J., Eghbali, N.: On approximate solutions of some delayed fractional differential equations. Appl. Math. Lett. 54, 31-35 (2016)

[2] Brzdek, J., Popa, D., Rasa, I.: Hyers Ulam stability with respect to gauges. J. Math. Anal. Appl. 453, 620-628 (2017)

[3] Brzdek, J., Popa, D., Raşa, I., Xu, B.: Ulam Stability of Operators. Academic Press, Oxford (2018)

[4] Diaz, J.B., Margolis, B.: A fixed point theorem of the alternative, for contractions on a generalized complete metric space. Bull. Am. Math. Soc. 74(2), 305-309 (1968)

[5] Diethelm, K., Ford, N.J.: Analysis of fractional differential equations. J. Math. Anal. Appl. 265, 229-248 (2002)

[6] Hyers, D.H.: On the stability of the linear functional equation. Proc. Nat. Acad. Sci. 27, 222-224 (1941)

[7] Hyers, D.H., Isac, G., Rassias, T.M.: Stability of Functional Equations in Several Variables. Birkhäuser, Boston (1998)

[8] Ibrahim, R.W.: Generalized Ulam-Hyers stability for fractional differential equations. Int. J. Math. 23(5), 9 (2012)

[9] Ibrahim, R.W.: Ulam stability for fractional differential equation in complex domain. Abstr. Appl. Anal. 2012, 1-8 (2012)

[10] Ibrahim, R.W.: Ulam-Hyers stability for Cauchy fractional differential equation in the unit disk. Abstr. Appl. Anal. 2012, 1-10 (2012)

[11] Jung, S.-M.: Hyers-Ulam-Rassias Stability of Functional Equations in Nonlinear Analysis. Springer, New York (2011)

[12] Killbas, A.A., Srivastava, H.M., Trujilo, J.J.: Theory and Applications of Fractional Differential Equations, North-Holland Mathematics Studies, vol. 204. Elsevier, Amsterdam (2006) 
[13] Li, Y., Wang, Y.: Uniform asymptotic stability of solutions of fractional functional differential equations. Abstr. Appl. Anal. 2013, 8 (2013)

[14] Mathai, A.M., Haubold, H.J.: Special Functions for Applied Scientists. Springer, Berlin (2008)

[15] Miller, K.S., Ross, B.: An Introduction to the Fractional Calculus and Differential Equations. Wiley, New York (1993)

[16] Miura, T.: On the Hyers-Ulam stability of a differentiable map. Sci. Math. Jpn. 55, 17-24 (2002)

[17] Miura, T., Takahasi, S.E., Choda, H.: On the Hyers-Ulam stability of real continuous function valued differentiable map. Tokyo J. Math. 24, 467-476 (2001)

[18] Peng, S., Wang, J.R.: Existence and Ulam-Hyers stability of ODEs involving two Caputo fractional derivatives. Electron. J. Qual. Theory Differ. Equ. 48-54(52), 1-16 (2015)

[19] Podlubny, I.: Fractional Differential Equations. Academic Press, San Diago (1999)

[20] Srivastava, H.M., Tomovski, Z.: Fractional calculus with an integral operator containing a generalized Mittag-Leffler function in the kernel. Appl. Math. Comput. 211, 198-210 (2009)

[21] Tarasov, V.E.: Fractional Dynamic: Application of Fractional Calculus to Dynamics of Particles. Springer, Berlin (2010)

[22] Ulam, S.M.: A Collection of Mathematical Problems. Interscience Publishers, New York (1968)

[23] Wang, J.R., Li, X.: Ulam-Hyers stability of fractional Langevin equations. Appl. Math. Comput. 258, 72-83 (2015)

[24] Wang, J.R., Lin, Z.: Ulam's type stability of Hadamard type fractional integral equations. Filomat 28(7), 1323-1331 (2014)

[25] Wang, J.R., Lin, Z.: A class of impulsive nonautonomous differential equations and Ulam-Hyers-Rassias stability. Math. Methods Appl. Sci. 38(5), 865-880 (2015)

[26] Wang, J.R., Lv, L., Zhou, Y.: Ulam stability and data dependence for fractional differential equations with Caputo derivative. Electron. J. Qual. Theory Differ. Equ. 63, 1-10 (2011)

[27] Wang, J.R., Lv, L., Zhou, Y.: New concepts and results in stability of fractional differential equations. Commun. Nonlinear Sci. Numer. Simul. 17, 2530-2538 (2012)

[28] Wang, J.R., Zhang, Y.: Ulam-Hyers-Mittag-Leffler stability of fractional-order delay differential equations. Optim. J. Math. Program. Optim. Res. 63(8), 11811190 (2014)

[29] Wang, J.R., Zhou, Y., Feckan, M.: Nonlinear impulsive problems for fractional differential equations and Ulam stability. Appl. Math. Comput. 64, 3389-3405 (2012)

[30] Wang, J.R., Zhou, Y., Lin, Z.: On a new class of impulsive fractional differential equations. Appl. Math. Comput. 242, 649-657 (2014) 
Janusz Brzdek

Faculty of Applied Mathematics

AGH University of Science and Technology

Mickiewicza 30

30-059 Kraków

Poland

e-mail: brzdek@agh.edu.pl

Nasrin Eghbali and Vida Kalvandi

Department of Mathematics with Applications, Faculty of Sciences

University of Mohaghegh Ardabili

Ardabil 56199-11367

Iran

e-mail: eghbali@uma.ac.ir;

vida.kalvandi@yahoo.com

Received: June 29, 2021.

Accepted: November 3, 2021.

Publisher's Note Springer Nature remains neutral with regard to jurisdictional claims in published maps and institutional affiliations. 